\title{
Hidden in plain sight: uncovering the role of CREB1 in HIV-1 vaccine-induced immunity
}

\author{
Systems approaches applied to human and nonhuman primate cohorts revealed that the transcription factor \\ CREB1 (cAMP-responsive element-binding protein 1) may be a key driver of human immunodeficiency virus 1 \\ vaccine-induced immunity.
}

\author{
Helder I. Nakaya
}

$\Lambda$ mong all human transcription factors, CREB1 (cAMP-responsive element-binding protein 1 ) is one of the best understood. Yet, Tomalka et al. ${ }^{1}$ have managed to uncover a hidden role for CREB1 in human immunodeficiency virus 1 (HIV-1) vaccine-induced immunity. Using a systems approach that combined different -omics technologies and three independent cohorts, they discovered that activation of CREB1 may drive the efficacy of vaccination against HIV-1 in nonhuman primates (NHPs), and also reduce the risk of viral acquisition in humans (Fig. 1).

In the year that saw the advent of coronavirus disease 2019 (COVID-19), another, much older, pandemic resulted in the death of more than 500,000 people worldwide: the HIV and AIDS pandemic (https://www.who.int/news-room/ fact-sheets/detail/hiv-aids). Vaccines represent potent weapons to combat this pandemic, but the vaccine trial with the most successful results so far achieved only around 30\% efficacy against HIV-1 infection ${ }^{2}$. In this human trial, known as the RV144 trial, over 16,000 volunteers received an ALVAC-HIV (vCP1521) and AIDSVAX $\mathrm{B} / \mathrm{E}$ vaccine regimen and were monitored for 3 years after vaccination ${ }^{2}$. Subsequent studies in humans and NHPs found that higher levels of IgG variable regions 1 and 2 (V1V2) antibodies to HIV-1 correlated with decreased risk of infection with HIV-1 or simian immunodeficiency virus (SIV $)^{3}$. These antibodies bind to the V1V2 of HIV-1 envelope (Env) proteins.

Systems vaccinology provides the perfect framework for bridging the gaps between vaccine clinical trials and discovery-based science ${ }^{4}$. By perturbing the biological system (that is, by vaccinating humans) and measuring responses using high-throughput technologies, researchers can create computational and mathematical models that may be used to predict or understand the immune responses

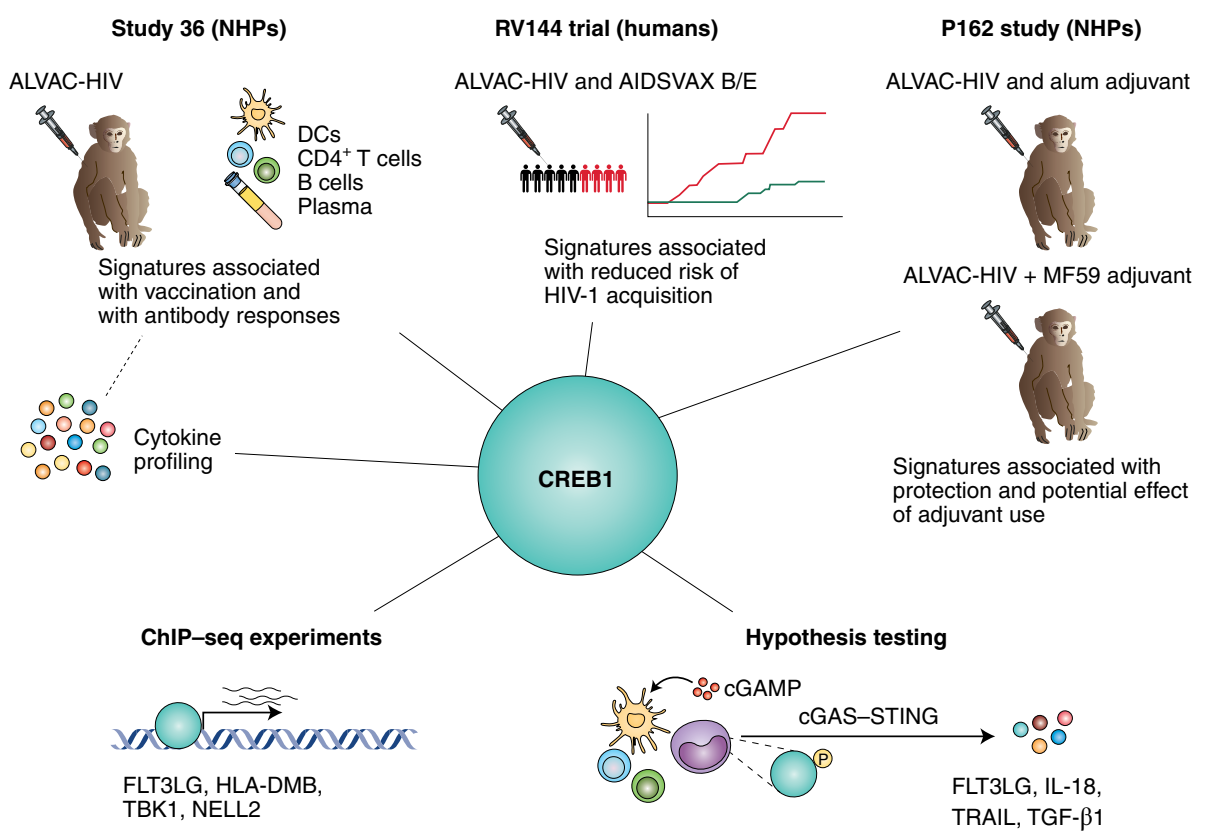

Fig. 1 | Systems vaccinology framework applied to HIV-1 vaccine. Tomalka et al ${ }^{1}$ combined different -omics technologies and three independent cohorts (study 36, RV144 trial and P162 study) to uncover a central role for CREB1 and its target genes in HIV-1 vaccine-induced immunity. DCs, dendritic cells.

induced by vaccination. Insights and hypotheses can then be generated from these models, which are later validated experimentally. Such frameworks have already been applied successfully to study a broad range of vaccines, from yellow fever ${ }^{5}$ to COVID- $19^{6}$ vaccines.

The work of Tomalka et al. ${ }^{1}$ now provides a great example of how systems vaccinology can be applied to HIV-1 vaccines.

Tomalka et al. ${ }^{1}$ integrated and analyzed high-throughput data collected from four independent cohorts to generate new mechanistic hypotheses that were later tested and confirmed by in vitro experiments. Initially, Tomalka et al. ${ }^{1}$ performed an immunogenicity study in NHPs to identify the transcriptomic signatures of isolated dendritic cells (DCs), CD4 $4^{+} \mathrm{T}$ cells and B cells that were induced by immunization with ALVAC and/or associated with titers of antibodies to V1V2s. Among these signatures, they found that the target genes of the transcription factor CREB1 were induced after vaccination with ALVAC and also correlated with V1V2 in all three immune cell subsets. This pointed to CREB1 as a putative driver of ALVAC-induced immunity. By reanalyzing microarray data from the RV144 case-control study ${ }^{7}$, which profiled 31 breakthrough cases and 151 uninfected vaccinees, Tomalka et al. ${ }^{1}$ confirmed, now in humans, that CREB1 target genes were induced by ALVAC and 
that their expression was correlated with levels of antibodies to V1V2s.

CREB1 is one of the most well-studied transcription factors, and modulates the expression of many target genes. A diverse array of receptors (including pattern recognition receptors and ligand-gated cation channel nucleotide receptors) can trigger the activation of kinases that will phosphorylate CREB1 inside the cell nucleus ${ }^{8}$. Once CREB1 is activated, it binds to DNA response elements known as cAMP-responsive transcriptional enhancer and recruits the CREB-binding protein, which initiates or represses the transcription of target genes. CREB1 has crucial roles in a variety of processes, from neuronal plasticity to cancer. Some of these roles are related to immune function, such as modulating nuclear factor kappa B and interferon gamma signaling, promoting the survival of macrophages and regulating $\mathrm{T}$ and $\mathrm{B}$ cells ${ }^{8}$.

There are thousands of putative CREB1 targets, including several cytokines and chemokines and their corresponding receptors. An interesting question is which of these targets is associated with reduced risk of HIV-1 acquisition in the RV144 trial. To address this question, the authors first split the participants on the basis of the increase in expression levels of CREB1 target genes after vaccination. They noticed that vaccinees with the highest induction of CREB1 target genes had a much lower risk of HIV-1 acquisition $(P<0.0001)$ than that of vaccinees who showed low induction of CREB1 target genes. Using an independent NHP study, the authors also demonstrated that the expression of several of those genes was induced in peripheral blood mononuclear cells as early as $24 \mathrm{~h}$ after vaccination (and persisted for up to 3 days) ${ }^{1}$.

To confirm that CREB1 bound directly to the promoter regions of key genes after stimulation with ALVAC, the authors performed chromatin immunoprecipitation followed by high-throughput sequencing experiments in NHP cells and human cells ${ }^{1}$. A strong overlap was seen between the genes induced by vaccination in the cohorts and the genes whose promoter regions CREB1 was bound to. The overlap includes genes encoding the signaling regulators CX3CL1 (fractalkine), CCL2 (MCP-1) and MAPK.

Among the ALVAC-induced CREB1 target genes, more than 100 were known mediators of immunity and inflammation. The authors used a custom array to confirm that the expression of several cytokines and chemokines was indeed induced in the plasma of NHPs after ALVAC immunization. Additional analyses showed that the plasma levels of CREB1-associated cytokines and chemokines, such as CX3CL1, TGF- $\beta 3$, CCL26 (eotaxin-3), IL-13 and CXCL11 (I-TAC), can predict the magnitude of the responses to antibodies to V1V2 ${ }^{1}$. Furthermore, Tomalka et al. ${ }^{1}$ assessed whether some of these vaccine-induced cytokines or chemokines were also associated with protection against infection. For this, they used blood transcriptome data from another vaccine challenge study, in which 54 rhesus macaques were primed with ALVAC-SIV alone, and boosted twice with ALVAC-SIV and gp120 in either MF59 adjuvant or alum adjuvant ${ }^{9}$. Among their findings, it was shown that plasma levels of the chemokine fractalkine, which promotes the survival and migration of human monocytes, was correlated positively with the number of challenges needed to infect NHPs. Notably, levels of CCL26, which may act as an antagonist of the chemokine receptor CCR2 and repress monocyte migration, were associated negatively with protection against infection.

Another interesting finding in the NHP cohort was that an ALVAC adjuvanted with the MF59 regimen did not induce expression of CREB1 target genes in the same way the ALVAC adjuvanted with alum $\mathrm{did}^{1}$. It is tempting to connect this finding with the differences in vaccine efficacy seen in the RV144 trial (which used alum as an adjuvant) and the South African HVTN702 trial (which used MF59 as an adjuvant) ${ }^{10}$. Although the vaccine regimen of the RV144 trial showed modest efficacy ${ }^{2}$, the vaccine regimen of the HVTN702 trial was unable to prevent HIV-1 infection ${ }^{10}$. Notably, there were several differences between the RV144 and HVTN702 trials, including other components of the vaccine regimen and even the differing prevalence of human leukocyte antigen genotypes in South African and Thai populations ${ }^{10}$.

In the quest for a mechanism that explains ALVAC-mediated immunity, the authors combined functional analyses and network analyses to identify central genes encoding molecules that are involved with specific immune functions ${ }^{1}$. TMEM173 (which encodes STING) was one of these central genes. Cytosolic DNA (including the DNA derived from HIV while infecting a cell) is sensed by cGAS, which then activates STING by the production of cGAMP. The authors hypothesized that both ALVAC and HIV could activate the cGAS-STING pathway in DCs, leading to CREB1 activation and the release of cGAMP.
The cGAMP released from DCs could then induce activation of CREB1 in other cell subsets, such as $\mathrm{CD} 4^{+} \mathrm{T}$ cells and B cells. They tested this hypothesis by stimulating human peripheral blood mononuclear cells with cGAMP and quantifying the phosphorylation of CREB1 in different subsets of immune cells. A rapid increase in phosphorylated CREB1 was observed in CD $4^{+} \mathrm{T}$ cells, B cells and monocytes or DCs. In $\mathrm{CD}^{+} \mathrm{T}$ cells and DCs, the stimulation of cGAMP induced the production of IL-18 and FLT3LG - two key cytokines whose levels correlated with titers of antibodies to V1V2 in the NHP study.

Back in 2012, we envisioned that systems vaccinology could affect the development of HIV vaccines ${ }^{11}$. Two ways that this could be achieved were: (1) unraveling new molecular insights about vaccine-induced immunity, and (2) identifying correlates of protection that were based on gene expression ${ }^{11}$. Tomalka et al. ${ }^{1}$ advanced on both of these fronts. Their work can guide the development and use of vaccine components or adjuvants that trigger the immune pathways associated with reducing HIV-1 acquisition. In addition, this work suggests that key CREB1 target genes and CREB1-associated cytokines or chemokines can be used as early correlates of immunogenicity or protection for HIV vaccines.

\section{Helder I. Nakaya (D) 1,2,3凶}

${ }^{1}$ Department of Clinical and Toxicological Analyses, School of Pharmaceutical Sciences, University of São Paulo, São Paulo, Brazil. ${ }^{2}$ Scientific Platform Pasteur-University of São Paulo, São Paulo,Brazil. ${ }^{3}$ Hospital Israelita Albert Einstein, São Paulo, Brazil. $凶_{e-m a i l: h e l d e r . n a k a y a @ e i n s t e i n . b r}$

Published online: 23 September 2021 https://doi.org/10.1038/s41590-021-01018-9

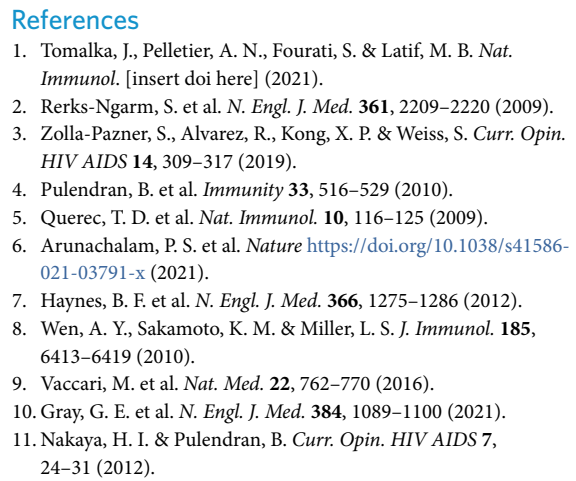

The author declares no competing interests. 